\title{
Monitoring Complete Blood Counts and Haemoglobin Levels in Osteoarthritis Patients: Results from a European Survey Investigating Primary Care Physician Behaviours and Understanding
}

\author{
Chris Walker ${ }^{*}, 1$, Augusto Faustino ${ }^{2}$ and Angel Lanas ${ }^{3}$ \\ ${ }^{1}$ Pfizer Ltd, Walton on-the-Hill, Tadworth, Surrey, UK \\ ${ }^{2}$ Clínica de Reumatologia de Lisboa, Lisboa, Portugal; Instituto Português de Reumatologia de Lisboa, Lisboa, \\ Portugal \\ ${ }^{3}$ University of Zaragoza, Aragón Health Research Institute (IIS Aragón), CIBERehd, Zaragoza, Spain
}

\begin{abstract}
Background: Chronic use of non-steroidal anti-inflammatory drugs (NSAIDs) is associated with an increased risk of gastrointestinal (GI) toxicity, including occult blood loss and the development of clinically significant anaemia.

Methods: 700 primary care physicians who routinely used NSAIDs to manage their patients were questioned to probe their understanding of the potential importance of blood loss in the OA populations they commonly treated with NSAIDs in a chronic fashion.

Results: Approximately $50 \%$ of doctors surveyed measured their osteoarthritis patients' haemoglobin routinely as part of a complete blood count (CBC). The remaining cohort of physicians only considered conducting CBCs if they believed there was cause for concern, with the most common reasons cited being anaemia/blood loss $(90 / 80 \%$ of physicians respectively) or the patient showing signs of weakness and fatigue ( $78 \%$ of physicians).

When all doctors were queried on their understanding of normal range of haemoglobin $(\mathrm{Hb})$ values, as defined by the WHO, significant variation in the absolute figures were reported with approximately $40 \%$ of physicians citing a low end range for normal that would actually place the patient below the threshold for anaemia.

Conclusion: Physician practice in relation to carrying out blood tests in OA patients and their understanding of the potential significance of specific results obtained, namely haemoglobin values, varies substantially across the countries surveyed. As NSAIDs form a pivotal part in the chronic treatment of osteoarthritis and are well recognised agents that can precipitate blood loss, guidelines may be needed to advise physicians as to when monitoring a patient's haemoglobin levels may be appropriate.
\end{abstract}

Keywords: Blood loss, NSAIDs, GI, osteoarthritis.

\section{INTRODUCTION: OSTEOARTHRITIS (OA) - A HIDDEN PROBLEM OF DISEASE MANAGEMENT WITH NON- STEROIDAL ANTI-INFLAMMATORY DRUGS?}

Worldwide, there are 135 million people who have OA, and it is becoming increasingly common with the aging population seen in most western societies [1]. Consequently, $\mathrm{OA}$ is the most frequent cause of disability in older age groups [2] and ranks among the top 10 causes of disability [3]. OA affects twice the number of women (18\%) than men $(9.6 \%)$ aged $\geq 60$ years [2]. NSAIDs are widely prescribed for long-term pain relief and inflammation in arthritis, with guidelines recommending their use after failure with paracetamol $[4,5]$. The focus of preventing GI damage with NSAIDs has, until now, been on the upper GI tract. Guidelines recommend the use of a ns-NSAID plus a gastroprotective agent (such as a PPI) or a cyclooxygenase

*Address correspondence to this author at the Pfizer Ltd., Walton on-theHill, Tadworth, Surrey, UK; Tel: 61737371031;

E-mail: chris.walker@pfizer.com
(COX)-2 selective inhibitor alone for patients at increased GI risk $[4,5]$ with one of the key factors identifying a patient as being at increased risk due to age $(>65)[6]$.

Large trials investigating the gastrointestinal toxicity of NSAIDs do however, suggest that $40-60 \%$ of serious GI toxicity may be in the lower GI tract $[7,8]$ with large scale epidemiological work $[9,10]$ and reviews of clinical trial work $[11,12]$ supporting this picture.

In the lower GI tract, it is proposed that NSAIDs induce changes in local prostaglandin metabolism, together with a topical toxic effect, that may be increased by enterohepatic circulation of the drug $[13,14]$. Mucosal cell integrity is compromised as a result, with increases in epithelial permeability allowing bacteria, toxins and bile acids to cause inflammation [15]. The inflammation commonly precipitates subclinical mucosal damage in the distal GI tract, and at least $60-70 \%$ of patients taking NSAIDs develop enteropathy [15, $16]$, which is associated with continuous and mild bleeding. This may in turn result in anaemia, or more obvious clinical damage to the lower bowel (ulcers and bleeds). 
The CONDOR (Celecoxib versus Omeprazole aNd Diclofenac for Osteoarthritis and Rheumatoid arthritis patients at increased GI risk) study, published in 2010 was the first large (more than 4,400 arthritis patients at increased GI risk), randomised GI outcomes study to investigate the potential frequency of defined amounts of blood loss $[(\geq 2 \mathrm{~g} / \mathrm{dL})$ decreases in haemoglobin (hb)] throughout the gastrointestinal tract, in addition to more well recognised clinical endpoints (perforations, obstructions and bleeds) [17]. Use of $\mathrm{a} \geq 2 \mathrm{~g} / \mathrm{dL}$ drop in haemoglobin has been well recognised as a surrogate endpoint in clinical trials investigating the gastrointestinal toxicity of NSAIDs conducted over the last twenty years [17-20].

The trial demonstrated a 4-fold superior risk reduction of clinically significant upper and lower GI events for arthritis patients taking celecoxib alone compared to diclofenac plus omeprazole. Differences were mainly attributable to the frequency of $\mathrm{Hb}$ decrease, with fewer patients in the celecoxib group having a significant decrease in $\mathrm{Hb}(2 \mathrm{~g} / \mathrm{dL}$ or more) than in the diclofenac plus omeprazole group (15 vs 77). The majority of these $\mathrm{Hb}$ drops were adjudicated as being of presumed occult GI sources. The study reported that the predefined $\mathrm{Hb}$ drop occurred as early as one to two months after starting NSAID treatment in some patients. Of the 92 patients who had a decrease of $2 \mathrm{~g} / \mathrm{dL}$ in $\mathrm{Hb}, 50$ had haemoglobin concentrations lower than $11.5 \mathrm{~g} / \mathrm{dL}$. (N.B $11.5 \mathrm{~g} / \mathrm{dL}$ was the central labs definition of anaemia for both sexes - and the most conservative estimation of the incidence of 'true' anaemia reported in the study)[17].

Whilst there is some contention in the literature [21,22] most observational cohorts adopt the World Health Organisation (WHO) criteria for anaemia - namely - a $\mathrm{Hb}$ concentration $<13 \mathrm{~g} / \mathrm{dL}$ in men or $<12 \mathrm{~g} / \mathrm{dL}$ in women. Evidence using these values suggests that anaemia is associated with poorer physical performance and greater disability [23], a doubling in the risk of recurrent falls [24], and a greater risk of mortality [25] compared to respective controls with non anaemic $\mathrm{Hb}$ levels.

Epidemiological studies have also shown that mildly low or low to normal $\mathrm{Hb}$ concentrations that do not meet the definition for anaemia are independently associated with an increased risk of frailty, poor functional outcomes, admission to hospital and mortality [26-28].

The questionnaire described below was designed with the intention of developing an initial understanding of European primary care physician practice for the collection of a complete blood counts (CBC) in osteoarthritis patients. A key goal was to understand how physicians interpret the information obtained, particularly in relation to the parameter of haemoglobin levels as an indicator of patient health (especially patient status as being anaemic/non-anaemic as defined by the WHO). Given that NSAIDs, including COX-2 selective inhibitors have been shown to have a strong causal association with blood loss from the gastrointestinal tract, it was felt that a snapshot survey would be useful to make an initial assessment of primary care physician awareness on this important topic.

\section{METHODS}

The aim of this survey was to provide an initial semiquantitative view of self-reported physician behaviour in relation to the practice of taking $\mathrm{CBC}$ 's and in particular haemoglobin values for patients they are treating who have osteoarthritis. The physicians were screened and excluded using the criteria below with the broad aim of the research being to identify a cohort of physicians who treated osteoarthritis frequently.

Physicians were excluded if they had practiced medicine for $<3$ or $>30$ years, were employed in any capacity by a pharmaceutical company/Clinical Research Organisation, saw fewer than 7 OA patients per month, or did not recognise themselves as being involved in the initiation or switching of pain medications for the treatment of osteoarthritis. In addition, physicians who self-recognised as prescribing less than 7 prescriptions for a COX-2 selective inhibitor (e.g. etoricoxib/celecoxib) were also de-selected from further follow up.

All information presented was gathered through the form of a short online questionnaire with doctors from 8 European countries (Belgium, France, Germany, Italy, Portugal, Spain, Switzerland, and UK) who were self-identified as working in a primary care environment. The exception was Portugal where the interviews were conducted face to face. One hundred physicians were questioned from France, Germany, Italy, Portugal, Spain, and the UK, with 50 physicians sampled from Switzerland and Belgium. All interviews took place in November and December 2009. The interviews were designed to be as short as possible (no interview/online interaction lasted greater than 15 minutes), and no physician demographics were captured other than the screener questions designed to assess their suitability for entry into the survey.

Physicians meeting the criteria above were asked the questions presented in the two exhibits below:

Exhibit 1. Questions on complete blood counts.

1) When if at all, do you undertake a complete blood count (CBC)
Choose the most appropriate answer; when needed (e.g. ad
hoc)/routinely/never
2) (If the physician answered that he or she undertook routine blood
tests) - please define your answer to the question routinely with one
of the following answers.
At least monthly/ every 2 months/ every $3-4$ months/ every $5-6$ months/
every $6-12$ months/ every $1-2$ years/ every $2-3$ years/ less than every 3
years.
3) (For Physicians answering on an ad hoc/when needed basis) What
would trigger you to undertake a complete blood count (CBC) if the
answer to question one was - when needed? Select all that apply
Occurrence of $\quad$ GI $\quad$ symptoms/suspected
loss/weakness/fatigue symptoms/blood loss/ nothing - they aren't
appropriate for this patient group/ other (please specify)
4) (For Physicians answering routinely) - For what reasons do you
undertake a complete blood count (CBC) routinely?
Select all that apply
a) To assess the absolute haemoglobin (Hb) and/or haematocrit (Hct)
levels of the patient on that day
b) To compare and assess changes in haemoglobin (Hb) and/or
haematocrit (Hct) levels over time by comparing results to previous
results
c) Follow guidelines
d) Personal preference or habit
e) Other.

Choose the most appropriate answer; when needed (e.g. ad hoc)/routinely/never

2) (If the physician answered that he or she undertook routine blood tests) - please define your answer to the question routinely with one of the following answers.

At least monthly/ every 2 months/ every 3-4 months/ every 5-6 months/ years.

3) (For Physicians answering on an ad hoc/when needed basis) What would trigger you to undertake a complete blood count $(\mathrm{CBC})$ if the answer to question one was - when needed? Select all that apply appropriate for this patient group/ other (please specify)

4) (For Physicians answering routinely) - For what reasons do you undertake a complete blood count (CBC) routinely?

a) To assess the absolute haemoglobin ( $\mathrm{Hb}$ ) and/or haematocrit (Hct) levels of the patient on that day

b) To compare and assess changes in haematocrit (Hct) levels over time by comparing results to previous results

e) Other. 
Exhibit 2. Questions on normal haemoglobin levels and the health/protection of the GI tract.

5) To the best of your knowledge, how would you define the normal haemoglobin $\mathrm{Hb}$ range for:

MALE PATIENT: (minimum $\mathrm{Hb}$ normal level g/dl: Maximum $\mathrm{Hb}$ normal level $\mathrm{g} / \mathrm{dl})$

FEMALE PATIENT (Not pregnant): (minimum $\mathrm{Hb}$ normal level $\mathrm{g} / \mathrm{dl}:$

Maximum $\mathrm{Hb}$ normal leve $\mathrm{g} / \mathrm{dl})$

6) How much of a decrease in haemoglobin $(\mathrm{Hb})$ would you consider clinically significant for you to take action?

Please answer in $\mathrm{g} / \mathrm{dl}$ (free text answer)

7) To what level would a patient's haemoglobin levels have to decrease for you to consider it clinically significant and to take action?

MALE PATIENT

Haemoglobin level for a male patient was originally [INSERT MAXIMUM Hb NORMAL LEVEL FROM Q5]: A decrease to $\mathrm{g} / \mathrm{dl}$ would be

clinically significant

Haemoglobin level for a male patient was originally [INSERT MAXIMUM $\mathrm{Hb}$ NORMAL LEVEL FROM Q5]: A decrease to

clinically significant

FEMALE PATIENT

Haemoglobin level for a female patient was originally [INSERT MAXIMUM Hb NORMAL LEVEL FROM Q5]: A decrease to would be clinically significant

Haemoglobin level for a female patient was originally [INSERT MAXIMUM Hb NORMAL LEVEL FROM Q5]: A decrease to $\mathrm{g} / \mathrm{dl}$ would be clinically significant

8) What is your level of concern with the GI tract of your OA patients?

Please give an answer for each of the following: upper, lower and entire GI tract. Rate on a scale from 1 to 10 where 1 is "not at all concerned" and 10 is "very concerned" (physicians who answered 8, 9 or 10 were viewed as having a high level of concern).

9) How would you rate the level of GI protection offered by PPIs?

Please give an answer for each of the following: upper, lower and entire GI tract. Please rate on a scale from 1 to 10 where 1 is "no protection at all" and 10 is "very high level of protection"(physicians who answered 8,9 or 10 were viewed as having a high level of concern).

\section{RESULTS: DO EUROPEAN PHYSICIANS CURREN- TLY CONDUCT COMPLETE BLOOD COUNTS IN (CBCS) OA PATIENTS?}

Approximately $50 \%$ of the 700 physicians surveyed suggested that OA patients would have a complete blood count (CBC) 'routinely' conducted with the percentage varying from $32 \%$ of Swiss doctors, to $64 \%$ of Italian doctors (see Table 1 for full country by country variations).

The most frequently observed definition of routine were based on a periodicity of every 6-12 months (39\% of physicians sampled - see Table 2 for full description of variations in periodicity) or every 5-6 months $(22 \%$ of physicians sampled).

The most common reasons (detailed data not shown) for conducting routine measurements were to compare to a previous measurement in the same patient (59\% of doctors) and to assess the absolute haemoglobin $(\mathrm{Hb})$ level at the time of the test (30\% of doctors). Personal preference was cited by $28 \%$ of physicians, with following guidelines (type of guidelines not specified) cited by $18 \%$ of doctors.

In the other group of approximately $50 \%$ of physicians that only conducted a CBC on an 'ad hoc' basis, for $90 \%$ a key potential reason cited was suspected anaemia in the patient concerned, with physicians also being concerned by blood loss ( $80 \%$ of physicians sampled) or by the patient showing signs of fatigue or weakness $(78 \%$ of physicians sampled).

\section{DEFINING THRESHOLDS FOR ANAEMIA}

Considering the entire cohort of doctors, and asking the question 'what constitutes the minimum and maximum normal range in haemoglobin values (in women and men)?' it was interesting to note that $46 \%$ of physicians cited a low end range value that would actually be an anaemic value for a male patient using the WHO criteria for anaemia. This figure was $41 \%$ in relation to a female population (data not shown).

A further question asking how much of a decrease in absolute haemoglobin values would trigger the physician to take action provided a very broad range of responses, with both small drops in haemoglobin $(1 \mathrm{~g} / \mathrm{dl})$ and large drops $(4 \mathrm{~g} / \mathrm{dl})$ in haemoglobin being fairly commonly cited. Interestingly, the measure commonly used in clinical trials considering NSAID safety (a $2 \mathrm{~g} / \mathrm{dl}$ drop in haemoglobin) was cited by $43 \%$ percent of physicians looking across the

Table 1. Thinking about your OA patients, when, if at all, do you undertake a complete blood count (CBC)? (possible answers: routinely/when needed/never).

\begin{tabular}{|c|c|c|c|}
\hline Country/Cohort Considered & Routine Examinations \% & Only when Needed \% & Never \% \\
\hline \hline Total Cohort & $\mathbf{4 9}$ & $\mathbf{4 9}$ & $\mathbf{2}$ \\
\hline France & 45 & 41 & 3 \\
\hline Germany & 53 & 35 & 1 \\
\hline Italy & 64 & 48 & 5 \\
\hline Spain & 52 & 66 & 3 \\
\hline UK & 41 & 52 & 2 \\
\hline Switzerland & 32 & 45 & 2 \\
\hline Belgium & 38 & 53 & \\
\hline Portugal & 53 & & \\
\hline
\end{tabular}

Survey answers based on a total sample of $n=704$ primary care physicians. Sample of approximately 100 physicians from all markets except Switzerland and Belgium (sample size approximately 50 physicians). 
entire sample with the figure in each respective country being as high as 54 and $56 \%$ in Switzerland and Belgium respectively, and as low as $27 \%$ in Italy (see Table 3 ).

Table 2. With what frequency do you undertake a complete blood count routinely? (sample of 345 physicians).

\begin{tabular}{|c|c|}
\hline Frequency of Measurement of CBC & \% of Routine CBC Measurers \\
\hline \hline At least monthly & $2 \%$ \\
\hline Every 2 months & $6 \%$ \\
\hline Every 3-4 months & $18 \%$ \\
\hline Every 5-6 months & $22 \%$ \\
\hline Every 6-12 months & $39 \%$ \\
\hline Every 1-2 years & $13 \%$ \\
\hline Every 2-3 Years & $1 \%$ \\
\hline Less than every 3 years & $0 \%$ \\
\hline
\end{tabular}

Whilst the answer to this last question is dependent on the patient's baseline value, a picture is partially formed where physicians who are monitoring $\mathrm{Hb}$ levels without complete awareness of the absolute values the medical community would define as being 'normal', or the size of a drop in $\mathrm{Hb}$ that should trigger concern.

\section{UNDERSTANDING GASTROINTESTINAL TOXICITY AND THE FUNCTION OF GASTROPROTECTIVE AGENTS}

The survey also probed physicians preferences in terms of treatment options for their osteoarthritis patients, and the associated concerns of treatment with non-steroidal treatments particularly, in terms of the upper and lower gastrointestinal tract. Results from the cohort overall (see Table 4 below) revealed that two-thirds $(65 \%)$ of physicians taking part were concerned (score 8-10) about the risks to the upper gastrointestinal tract whilst the figure was only $39 \%$ showing the same level of concern for the lower GI tract. A picture was consistently seen across all 8 markets, with the comparative difference in the level of concern between upper and lower GI tract being as high as 38\% in Switzerland and as low as $15 \%$ in Spain. It is notable that for either part of the gastrointestinal tract the level of concern was significantly lower in France than for any other market.

For the final question rating the level of protection offered by proton pump inhibitors the majority of physicians were aware that a proton pump inhibitor (PPI), only protects the upper GI tract: it is of concern, however that $21 \%$ mistakenly think protection extends to the lower GI tract.

Again there were substantial differences between countries with the percentage of physicians who answered this question incorrectly being as low as $12 \%$ in Belgium and Switzerland, but as high as $32 \%$ in Spain and $43 \%$ in Portugal (see Table 5 for full country analysis).

\section{DISCUSSION}

The survey of doctors from across Western Europe reported here, suggests there is insufficient awareness of the need to monitor for NSAID-related blood loss, potentially increasing the risk of patients suffering GI problems (anaemia and other clinical manifestations of harm). The inclusion criteria for this survey were designed to capture the views of doctors with at least a reasonable degree of familiarity of the classes of medicine under investigation and their associated toxicities, perhaps suggesting that had the inclusion criteria being less exacting the findings would have been even more marked. Whilst the findings are only intended to provide a spontaneous snapshot of physician practice and understanding, the sample is of a reasonable size, and is the first attempt, to the authors' knowledge, at investigating the views of primary care physicians from across several European countries. Given that OA is a condition primarily treated by this physician group the findings are important, and highlight that more could be done to raise awareness of the importance of $\mathrm{Hb}$ as a haematological parameter that may be altered unfavourably by medicines used to treat the condition.

The survey does have weaknesses. It lacks the benefit of a more formalised design, where information would be independently verified rather than just reported by the physician questioned. Capturing a greater quality and quantity of information on physician and patient phenotypes would allow better interrogation of some of the differences tentatively identified here, including the high awareness and

Table 3. How much of a decrease in haemoglobin (Hb) would you consider clinically significant for you to take action? ( $\mathrm{n}=704)$.

\begin{tabular}{|c|c|c|c|}
\hline Country/Cohort Considered & $\mathbf{2 g} / \mathbf{d l}$ Drop in Hb & $\mathbf{2 g} / \mathbf{d l}$ Drop in Hb & $\mathbf{2 g} / \mathbf{d l}$ Drop in Hb \\
\hline \hline Total Cohort & $\mathbf{2 3}$ & $\mathbf{4 3}$ & $\mathbf{3 4}$ \\
\hline France & 18 & 41 & 32 \\
\hline Germany & 25 & 43 & 63 \\
\hline Italy & 10 & 27 & 48 \\
\hline Spain & 14 & 44 & 20 \\
\hline UK & 36 & 54 & 14 \\
\hline Switzerland & 32 & 56 & 26 \\
\hline Belgium & 18 & 50 & 14 \\
\hline Portugal & 36 & & \\
\hline
\end{tabular}


concern with lower GI toxicity observed in Spain and Portugal (answer to question 4), that appears to be matched by an erroneous belief that proton pump inhibitors mitigate the problem (see question 5).

Table 4. What is your level of concern with the GI tract of your OA/RA patients? Rate on a scale from 1 to 10 where 1 is "not at all concerned" and 10 is "very concerned" Base: All respondents $(n=704)$. A summary of those who scored an 8 or above is provided in the table below.

\begin{tabular}{|c|c|c|}
\hline Country/Cohort Considered & Upper GI Tract \% & Lower GI Tract \% \\
\hline \hline Total Cohort & $\mathbf{6 5}$ & $\mathbf{3 9}$ \\
\hline France & 33 & 16 \\
\hline Germany & 72 & 39 \\
\hline Italy & 70 & 31 \\
\hline Spain & 77 & 62 \\
\hline UK & 70 & 40 \\
\hline Switzerland & 60 & 22 \\
\hline Belgium & 48 & 28 \\
\hline Portugal & 77 & 59 \\
\hline
\end{tabular}

Survey answers based on a total sample of $n=704$ primary care physicians. Sample of approximately 100 physicians from all markets except Switzerland and Belgium (sample size approximately 50 physicians).

Table 5. How would you rate the level of protection offered by Proton Pump Inhibitors (PPIs) for the upper and lower GI tract where one is 'no protection at all' and 10 is 'very high level of protection. A summary of those who scored an 8 or above is provided in the table below.

\begin{tabular}{|c|c|c|}
\hline Country/Cohort Considered & Upper GI Tract \% & Lower GI Tract \% \\
\hline \hline Total Cohort & $\mathbf{6 8}$ & $\mathbf{2 1}$ \\
\hline France & 57 & 16 \\
\hline Germany & 66 & 14 \\
\hline Italy & 69 & 15 \\
\hline Spain & 78 & 32 \\
\hline UK & 59 & 14 \\
\hline Switzerland & 78 & 12 \\
\hline Belgium & 64 & 12 \\
\hline Portugal & 77 & 43 \\
\hline
\end{tabular}

Survey answers based on a total sample of $n=704$ primary care physicians. Sample of approximately 100 physicians from all markets except Switzerland and Belgium (sample size approximately 50 physicians).

A more detailed qualitative design may also help explain whether the relatively low level of concern illustrated by French primary care physicians (answers to question 4) is explained by genuine physician beliefs, or some form of existing general practice/guideline that physicians believe meet this area of medical need (and therefore mitigates concern). To date the authors are not aware of any guidelines for OA in the countries surveyed that recommend $\mathrm{Hb}$ monitoring.

\section{APPLYING THE EVIDENCE: WHAT'S NEXT?}

Results of the CONDOR trial suggest that in as many as $60 \%$ of patients experiencing a trial endpoint, the subtle manifestations of potential harm $(\geq 2 \mathrm{~g} / \mathrm{dl}$ drops in $\mathrm{Hb})$ are of occult GI origin, including presumed small bowel blood loss. The results of a second trial in over 8,000 North American patients with osteoarthritis are largely supportive of these findings [29]. Neither primary publication provided further information on specific risk factors associated with a $>2 \mathrm{~g} / \mathrm{dL}$ drop, however a post hoc review of the 3700 osteoarthritis patients from the CONDOR trial suggested that increased age (assessed as a continuous variable), a history of GI Intolerance, high BMI index (continuous variable), positive $\mathrm{H}$. pylori at screening and raised C-reactive protein were all significantly associated with a greater risk of an OA patient experiencing a 2 $\mathrm{g} / \mathrm{dL}$ drop in haemoglobin [30].

\section{IF GREATER ATTENTION SHOULD BE GIVEN TO THE LOWER GI TRACT IN OA PATIENTS ON NSAIDs, HOW SHOULD WE DO THIS IN PRACTICE?}

The post hoc study outlined above did not differentiate between the $>2 \mathrm{~g} / \mathrm{dL}$ drops of defined origin and occult origin and as such the risk factors for lower GI harm are still strictly speaking unidentified.

As this is the case, it is more difficult to advise how to minimise the potential lower gastrointestinal harm that may be experienced as a result of use of NSAIDs. More research is needed to identify the risk factors that are specifically linked to lower gastrointestinal harm. In the meantime, a pragmatic approach may be to check a patient's baseline $\mathrm{Hb}$ before starting chronic NSAID treatment, then measure after one month of treatment before monitoring every six months. The high prevalence of the disease could make this too onerous a step for every patient with OA. As increased age, (particularly beyond 60-65) has been identified as a gastrointestinal risk factor in the literature $[6,29]$ it may be possible to identify an age based cut off, with patients above a certain age being monitored as described. Given that even 'low normal' $\mathrm{Hb}$ levels (e.g. non anaemic) have been associated with unfavourable outcomes in patients aged 70-80 [26], it would seem important to pay particular attention to this growing sector of the population.

\section{ONCE A SIGNIFICANT HB REDUCTION HAS BEEN OBSERVED, WHAT ACTION SHOULD BE TAKEN?}

If patients have been on a PPI together with the NSAID, the probability of having lesions in the upper GI tract is low, but still possible, as has been shown in the CONDOR trial. In these circumstances an upper GI endoscopy may be advisable. A PPI should also be prescribed, if not done before, based on the presence of risk factors and endoscopy findings. Patients should be further investigated with colonoscopy if they are at risk for colon cancer. If NSAIDs are needed, management options include stopping NSAID treatment, switching to a selective COX-2 inhibitor, or using other treatment approaches.

\section{CONCLUSION}

Reducing the risk of bleeding throughout the entire GI tract in OA patients being treated with NSAIDs can improve 
the health of individual patients affected, and reduce expenditure on further investigations and care. The survey presented here suggests the potential for the more subtle forms of bleeding that may occur with NSAID use, particularly from the lower gastrointestinal tract, are an under recognised problem. Further research is still required to supplement the one study that begins to suggest what the risk factors for gastrointestinal harm are beyond the duodenum [30]. Given that $\mathrm{OA}$ is an increasingly prevalent disease in societies with aging populations it may also be time to develop guideline recommendations on monitoring $\mathrm{Hb}$ levels in the growing populations of elderly OA patients who use NSAIDs.

\section{CONFLICT OF INTEREST}

The authors confirm that this article content has no conflict of interest.

\section{ACKNOWLEDGEMENTS}

We would like to thank Dr Marianne Fletcher (conduct and initial presentation of market research findings) and $\mathrm{Dr}$ Margaret Essex (thoughtful review of the manuscript).

Funding of market research: Pfizer Ltd.

\section{REFERENCES}

[1] Bone and Joint Decade Report 2009. Made to Last: Looking Back at a Decade of Action in Musculoskeletal Health, 2009.

[2] Woolf AD, Pfleger B. Burden of major musculoskeletal conditions policy and practice. Bull WHO 2003; 81: 646-56.

[3] Mathers CD, Lopez AD, Murray CJL. Global burden of disease and risk factors. Washington, DC: Oxford University Press 2006.

[4] Jordan KM, Arden NK, Doherty M, et al. EULAR recommendations 2003: an evidence based approach to the management of knee osteoarthritis: Report of a task force of the standing committee for international clinical studies including therapeutic trials (ESCISIT). Ann Rheum Dis 2003; 62: 1145-55.

[5] Zhang W, Doherty M, Arden NK, et al. EULAR recommendations: report of a task force force of the standing committee for international clinical studies including therapeutic trials (ESCISIT). Ann Rheum Dis 2005; 64: 669-81.

[6] Gutthann SP, Rodriquez LAG, Raiford DS. Individual non steroidal anti-infllamatory drugs and other risk factors for upper gastrointestinal bleeding and perforation. Epidemiology 1997; 8: 18-24.

[7] Silverstein FE, Graham DY, Senior JR, et al. Misoprostol reduces serious gastrointestinal complications in patients with RA receiving non steroidal anti-inflammatory drugs. Ann Intern Med 1995; 123: 241-9.

[8] Laine L, Connors LG, Reicin A, et al. Serious lower gastrointestinal clinical events with non selective NSAID or coxib use. Gastroenterology 2003; 124: 288-92.

[9] Lanas A, Garcia-Rodriguez LA, Polo-Tomás M, et al. Time trends and impact of upper and lower gastrointestinal bleeding and perforation in clinical practice. Am J Gastroenterol 2009; 104: 1633-41.

[10] Zhao Y, Encinosa W. Hospitalisations for gastrointestinal bleeding in 1998 and 2006. Statistical Brief 65. December 2008. Agency for Healthcare Research Quality. Available at http://www.hcupus.ahrq.gov/reports/statsbriefs/sb65.pdf
[11] Laine L. GI risk and risk factors of NSAIDs. J Cardiovascular Pharmacol 2006; 47 (Suppl 1): S60-S66.

[12] Laine L, Smith R, Min K, Chen C, Dubois RW. Systematic review: the lower gastrointestinal adverse effects of non-steroidal anti-inflammatory drugs. Aliment Pharmacol Ther 2006; 24: 751-67.

[13] Bjarnason I, Fehilly B, Smethurst P, et al. Importance of local versus systemic effects of non-steroidal anti-inflammatory drugs in increasing small intestinal permeability in man. Gut 1991; 32: 275-7.

[14] Whittle BJR. Mechanisms underlying intestinal injury induced by antiinflammatory CO inhibitors. Eur J Pharmacol 2004; 500: 427-39.

[15] Sigthorsson G, Tibble J, Hayllar J, et al. Intestinal permeability and inflammation in patients on NSAIDs. Gut 1998; 43: 506-11.

[16] Adebayo D, Bjarnason I. Is non-steroidal anti-inflammatory drug (NSAID) enteropathy clinically more important than NSAID gastropathy. Postgrad Med J 2006; 82: 186-91.

[17] Chan FKL, Lanas A, Scheiman J, et al. Celecoxib versus omeprazole and diclofenac in patients with osteoarthritis and rheumatoid arthritis (CONDOR): a randomized trial. Lancet 2010; Published online June 17, 2010 DOI:10.1016/S0140-6736(10) 60673-3.

[18] Bombardier C, Laine L, Reicin A, et al. Comparison of upper GI toxicity of rofecoxib and naproxen for patients with rheumatoid arthritis. N Eng J Med 2000; 343: 1520-8.

[19] Schnitzer TJ, Burmester GR, Mysler E, et al. Comparison of lumiracoxib with naproxen and ibuprofen in the therapeutic arthritis research and gastrointestinal event trial (TARGET), reduction in ulcer complications: randomised controlled trial. Lancet 2004; 364(9435): 665-74.

[20] Chan FKL, Cryer B, Goldstein JL, et al. A novel composite endpoint to evaluate gastrointestinal (GI) effects of non-steroidal anti-inflammatory drugs through the entire GI tract. J Rheumatol 2009; 37 (12): 167-74.

[21] Gaskell H, Derry S, Moore RA, et al. Prevalance of anaemia in older persons; systematic review. BMC Geriatrics 8: 1 Available online at http://www.biomedcentral.com/1471-2318/8/1

[22] Nilsson-Ehle H, Jagerburg R, Landahl S, et al. Blood hamoglobin declines in the elderly: implications for reference intervals from age 7088. Eur J Haematol 2000; 65: 297-305.

[23] Penninx BWJH, Pahor M, Cesari M, et al. Anemia is associated with disability and decreased physical performance and muscle strength in the elderly. J Am Geriatr Soc 2004; 52: 719-24.

[24] Penninx BWJH, Pluijm SMF, Lips P, et al. Late-life anemia is associated with increased risk of recurrent falls. J Am Geriatr Soc 2005; 53: 2106-11.

[25] Zakai NA, Katz R, Hirsch C, et al. A prospective study of anemia status, hemoglobin concentration, and mortality in an elderly cohort. Arch Int Med 2005; 165: 2214-20.

[26] Chaves PHM, Ashar B, Guralnik JM, et al. Looking at the relationship between hemoglobin concentration and prevalent mobility difficulty in older women. Should the criteria currently used to define anemia in older people be reevaluated? J Am Geriatr Soc 2002; 50: 1257-64.

[27] Chaves PH, Semba RD, Leng SX, et al. Impact of anaemia and cardiovascular disease on frailty status of community-dwelling older women: The women's health and aging studies I and II. J Gerontol A Biol Sci Med Sci 2005; 60: 729-35.

[28] Chaves P, Xue QL, Guralnik JM, et al. What constitutes normal haemoglobin in community dwelling older women. J Am Geriatr Soc 2004; 52: 1811-6.

[29] Cryer B, Chunming L, Simon L, et al. GI-REASONS: a novel 6-month, prospective, randomised, open-label, blinded endpoint (PROBE) trial. Am J Gastroenterol 2013; 108: 392-400.

[30] Lanas A, Goldstein JL, Chan FKL, et al. Risk factors associated with a decrease $>2 \mathrm{~g} / \mathrm{dL}$ in haemoglobin and/or $>10 \%$ haematocrit in osteoarthritis patients taking celecoxib or a non selective NSAID plus a PPI in a large randomised controlled trial (CONDOR). Aliment Pharmacol Ther 2012; 36(5): 485-92. 